\title{
8 \\ Small sets of complementary observables
}

\author{
M. Grassl, ${ }^{1}$ D. McNulty, ${ }^{2}$ L. Mišta Jr., ${ }^{3}$ and T. Paterek ${ }^{4,5,6}$ \\ ${ }^{1}$ Max Planck Institute for the Science of Light, Erlangen 91058, Germany \\ ${ }^{2}$ Department of Applied Mathematics, Hanyang University (ERICA), 55 Hanyangdaehak-ro, Ansan, Gyeonggi-do 426-791, Korea \\ ${ }^{3}$ Department of Optics, Palacký University, 17 listopadu 12, Olomouc 771 46, Czech Republic \\ ${ }^{4}$ School of Physical and Mathematical Sciences, Nanyang Technological University, 637371, Singapore \\ ${ }^{5}$ Centre for Quantum Technologies, National University of Singapore, 117543, Singapore \\ ${ }^{6}$ MajuLab, CNRS-UNS-NUS-NTU International Joint Research Unit, UMI 3654, Singapore
}

(Received 27 November 2016; published 17 January 2017)

\begin{abstract}
Two observables are called complementary if preparing a physical object in an eigenstate of one of them yields a completely random result in a measurement of the other. We investigate small sets of complementary observables that cannot be extended by yet another complementary observable. We construct explicit examples of unextendible sets up to dimension 16 and conjecture certain small sets to be unextendible in higher dimensions. Our constructions provide three complementary measurements, only one observable away from the ultimate minimum of two. Almost all our examples in finite dimensions are useful for discriminating pure states from some mixed states, and they help to shed light on the complex topology of the Bloch space of higher-dimensional quantum systems.
\end{abstract}

DOI: 10.1103/PhysRevA.95.012118

\section{INTRODUCTION}

Quantum mechanics restricts precision with which certain (incompatible) observables can be measured, as characterized, e.g., by the Heisenberg uncertainty relation. Here we study complementary observables which are, per definition, the most incompatible ones. The idea is clearly illustrated if one considers sequential measurements of such observables. After measuring one of them and subjecting the resulting object to a measurement of any other complementary observable, the results are completely random and independent of the particular outcome observed in the first measurement. The eigenstates of such observables form so-called mutually unbiased bases (MUBs), and due to their elementary role in quantum physics they have received considerable attention [1]. Typically, researchers ask about the maximal number of complementary observables. It is known that the maximal possible number of $d+1$ of them for a $d$-level object can be reached when $d$ is a power of a prime. Compelling evidence suggests that the maximum is strictly smaller for other dimensions.

Here we focus on the opposite question: what is the minimal number of complementary measurements? It has been observed in various construction methods for MUBs that if one starts "wrongly", it is impossible to construct all $d+1$ complementary measurements; one gets stuck at some strictly smaller number [2-8]. Such sets of complementary observables are called unextendible, and they are known to exist in small dimensions as well as in all dimensions $p^{2}$, where $p$ is a prime satisfying $p \equiv 3 \bmod 4$ [9], and in all dimensions $2^{m}$ for even $m$ [10]. Other examples are known if the notion of unextendibility is somewhat relaxed [11-13]. From this perspective we are interested in small sets of unextendible MUBs.

Our results for small dimensions are summarized in Table I. The sets that we construct contain no more than three measurements. While we were not able to prove that they are the minimal sets, they contain only one more measurement than the ultimate minimum. ${ }^{1}$ Quite surprisingly this is also the case for certain prime dimensions where we provide explicit examples in small dimensions, and a candidate set for more general dimensions. We also discuss various approaches to unextendibility which reveal the existence of unextendible sets of complementary observables in infinitely many dimensions.

\section{PROBLEM AND SUMMARY}

Consider the scenario depicted in Fig. 1. We are given a sequence of $n$ players. Starting with a completely mixed state, player $j$ either performs the measurement $M_{j}$ or passes the state unperturbed to the next player. It is required that all the measurements have completely random outcomes, independent of the results of the previous measurement and which of the measurements have been performed. This is formally encoded by the property of mutual unbiasedness:

$$
\left|\left\langle k_{j} \mid k_{j^{\prime}}^{\prime}\right\rangle\right|^{2}=1 / d \text { for } j \neq j^{\prime},
$$

where $\left|k_{j}\right\rangle$ is the $k$ th state of the eigenbasis of the $j$ th observable. The question we investigate is how small can $n$ be, such that no further player could guarantee that the measurement he or she performs will possess random outcomes.

The results are presented in Table I, showing that the number $n$ of measurements is very small. Apart from fundamental interest, this also has practical applications in witnessing various physical properties. We briefly describe how the unextendible sets help in witnessing the degree of purity of a state.

A set of MUBs is called strongly unextendible if there is not even a single vector unbiased to all the vectors in the set. In this case there is not a single pure quantum state that gives rise to completely random results when measured

\footnotetext{
${ }^{1}$ There always exists at least one MUB with respect to a given basis [14].
} 
TABLE I. Summary of the results. Note the intriguing case of dimension 6 for which there exists a set with only two measurements. All the cardinalities starting with dimension 7 are derived here.

\begin{tabular}{lc}
\hline \hline Dimension & Cardinality of minimal set \\
\hline 2 & 3 \\
3 & 4 \\
4 & 3 \\
5 & 6 \\
6 & 2 \\
7 & $\leqslant 3$ \\
$\vdots$ & $\vdots$ \\
16 & $\leqslant 3$ \\
$\infty$ & 3 for observables $r(\cos \theta \hat{q}+\sin \theta \hat{p})$ \\
\hline \hline
\end{tabular}

with the observables from the unextendible set. In other words, the entropy of the measurement results summed over the observables has an upper bound $H_{\mathrm{p}}<n \log _{2} d$. Hence, observing larger entropy than $H_{\mathrm{p}}$ indicates that a mixed state is being measured. All our examples in Table I, except for $d=6$, form strongly unextendible MUBs. Note that any pair of MUBs can never be strongly unextendible since for any two orthonormal bases of a $d$-dimensional Hilbert space, there always exists a vector unbiased to both $[15,16]$.

We would also like to note that the existence of unextendible MUBs provides information on the complex topology of the "Bloch" space of quantum states of higher-dimensional quantum systems. A density matrix describing a pure state of a $d$-level quantum system can be written in the operator basis of generalized Pauli matrices [17,18]. In this Bloch representation a pure quantum state is mapped to a normalized vector with $d^{2}-1$ real coefficients. It turns out that the resulting set of states is rather complex for dimensions $d \geqslant 3$, and unextendible MUBs provide a hint for why this is the case. While mutually unbiased states are mapped to orthogonal Bloch vectors, orthogonal quantum states are mapped to nonorthogonal Bloch vectors with an angle $\cos \theta=-\frac{1}{d-1}$ between them. A basis in the Hilbert space translates to a regular $(d-1)$-dimensional simplex in the Bloch space [19].

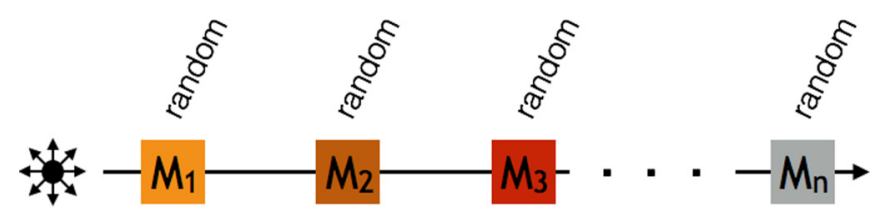

FIG. 1. The problem studied here can be visualized as follows. A physical system prepared initially in a completely mixed state is interrogated by a sequence of $n$ players. Each of them chooses to perform the corresponding projective measurement $M_{j}$ or forwards the system unperturbed to the next player. It is required that all the measurements have completely random outcomes, independent of the results of the previous measurement and which of the measurements have been performed. We ask about the smallest number of players such that no further player could guarantee that the measurement he or she performs will possess random outcomes, starting with a $d$-level input state. See Table I for a summary in small dimensions and the main text for other dimensions.
Thus the nonexistence of a vector that is unbiased to a set of MUBs means that there is not a single unit Bloch vector of a physical pure quantum state in the orthogonal complement to the space spanned by the Bloch vectors of unextendible MUBs. These orthogonal complements are of high dimensionality given by $d^{2}-1-m(d-1)$, where $m$ is the number of bases in the unextendible set. For example, in the Bloch space of a four-level system, there exists a 6-dimensional subspace (out of 15 dimensions in total) with no unit Bloch vector representing a physical pure state.

The paper is organized around Table I. In Sec. III we gather previous results from which one can infer the size of the minimal set of complementary observables up to dimension 6 (including 6). The new results in the Table are obtained by different methods which are described in subsequent sections. We first arrive at various candidate unextendible sets and next show that they are indeed unextendible by computer-aided algebraic methods. Finally, we discuss various approaches to unextendibility and use them to prove the existence of unextendible sets in many additional dimensions. ${ }^{2}$

\section{SMALL DIMENSIONS $(d \leqslant 6)$}

Let us begin by recalling the cardinalities of minimal sets of complementary observables in dimensions $d \leqslant 6$. If the standard basis is represented by the identity matrix, then the matrices representing all bases unbiased to it must have all entries of the same modulus. The results below follow from studies of these so-called complex Hadamard matrices. Note that two Hadamard matrices are equivalent if one transforms into the other by multiplication with permutation and diagonal matrices, and two sets of MUBs are equivalent if a unitary matrix maps one set to the other.

In dimension 2 we have no unextendible MUBs. All $2 \times 2$ Hadamard matrices are equivalent to the Fourier matrix $F_{2}$, and only one triple of MUBs exists. These three bases correspond to the eigenstates of the Pauli operators $\sigma_{x}, \sigma_{y}$, and $\sigma_{z}$.

Similarly, in dimension 3 no unextendible MUBs exist. All Hadamard matrices are equivalent to the Fourier matrix $F_{3}$, and six vectors are mutually unbiased to the pair $\left\{\mathbb{1}, F_{3}\right\}$. These six vectors can be partitioned into two bases. Together with $\mathbb{1}$ and $F_{3}$ they form a unique quadruple of MUBs that contains two inequivalent triples of MUBs.

The smallest case in which unextendible MUBs appear is dimension 4, where an infinite family of cardinality three exists. In this dimension all pairs of MUBs are equivalent to a member of the one-parameter set $\left\{\mathbb{1}, F_{4}(a)\right\}$, where $F_{4}(a)$ is the one-parameter Fourier family of $4 \times 4$ matrices. Each of these pairs extends to a mutually unbiased triple $\left\{\mathbb{1}, F_{4}(a), H_{2}(b, c)\right\}$, producing a three-parameter set. See Ref. [22] for the

\footnotetext{
${ }^{2}$ Note that the word "unextendible" in connection with bases in Hilbert spaces is sometimes used in the literature in a different context. An unextendible product basis, as in Ref. [20], denotes an incomplete orthogonal product basis whose complementary subspace contains no product state. Similarly, an unextendible maximally entangled basis, as in Ref. [21], denotes an incomplete set of orthonormal maximally entangled states which have no additional maximally entangled vectors orthogonal to all of them.
} 
definitions of $F_{4}(a)$ and $H_{2}(b, c)$. If $a=\pi / 2$, then unique sets of four and five MUBs exist (note that a set of $d$ MUBs in dimension $d$ is always extendible [23]), and all other values of $a$ give rise to an unextendible set. A simple example of an unextendible triple of MUBs is the following:

$$
\begin{aligned}
B_{1} & =\left(\begin{array}{llll}
1 & 0 & 0 & 0 \\
0 & 1 & 0 & 0 \\
0 & 0 & 1 & 0 \\
0 & 0 & 0 & 1
\end{array}\right), \quad B_{2}=\frac{1}{2}\left(\begin{array}{rrrr}
1 & 1 & 1 & 1 \\
1 & i & -1 & -i \\
1 & -1 & 1 & -1 \\
1 & -i & -1 & i
\end{array}\right), \\
B_{3} & =\frac{1}{2}\left(\begin{array}{cccc}
1 & e^{i a} & 1 & -e^{i a} \\
1 & -e^{i a} & 1 & e^{i a} \\
1 & e^{i b} & -1 & e^{i b} \\
1 & -e^{i b} & -1 & -e^{i b}
\end{array}\right),
\end{aligned}
$$

with $a, b \in[0, \pi), a \neq \pi / 2$, and where the rows of each matrix represent the orthogonal vectors of each basis.

In dimension 5 there are no unextendible MUBs. According to [24] there is a unique choice of Hadamard matrix, $F_{5}$, and exactly 20 vectors are mutually unbiased to the pair $\left\{\mathbb{1}, F_{5}\right\}$. These vectors can be partitioned into four orthonormal bases, resulting in a unique combination of six MUBs [22].

It turns out that our problem is also solved for dimension 6: there is a pair of complementary measurements which do not extend to a triple [5]. This pair corresponds to the set $\left\{\mathbb{1}, S_{6}\right\}$, where $S_{6}$ is the isolated Hadamard matrix [25]:

$$
S_{6}=\frac{1}{\sqrt{6}}\left(\begin{array}{cccccc}
1 & 1 & 1 & 1 & 1 & 1 \\
1 & 1 & \omega_{3} & \omega_{3} & \omega_{3}^{2} & \omega_{3}^{2} \\
1 & \omega_{3} & 1 & \omega_{3}^{2} & \omega_{3}^{2} & \omega_{3} \\
1 & \omega_{3} & \omega_{3}^{2} & 1 & \omega_{3} & \omega_{3}^{2} \\
1 & \omega_{3}^{2} & \omega_{3}^{2} & \omega_{3} & 1 & \omega_{3} \\
1 & \omega_{3}^{2} & \omega_{3} & \omega_{3}^{2} & \omega_{3} & 1
\end{array}\right)
$$

with $\omega_{3}=\exp (2 \pi i / 3)$. A complete classification of Hadamard matrices in dimension 6 is unknown, but other minimal sets may exist.

\section{DIMENSIONS 7, 11, AND PRIMES $p \equiv 3 \bmod 4$}

We begin with a lemma that holds in arbitrary dimensions. It will then be utilized in the construction of a particular triple of MUBs that will be shown to be unextendible by computeraided techniques.

The lemma utilizes the Fourier basis, which is defined as

$$
|\tilde{J}\rangle=F|j\rangle=\frac{1}{\sqrt{d}} \sum_{m=0}^{d-1} \omega_{d}^{j m}|m\rangle,
$$

where $\omega_{d}=\exp (2 \pi i / d)$ is a $d$ th root of unity, $\{|j\rangle\}$ denotes the standard basis, and $F$ denotes the Fourier matrix with elements $F_{j m}=\frac{1}{\sqrt{d}} \omega_{d}^{j m}$, where the rows and columns are enumerated as $j, m=0, \ldots, d-1$.

Lemma 1. Let $\left|v^{(0)}\right\rangle=\sum_{m=0}^{d-1} v_{m}|m\rangle$ be a state that is unbiased with respect to the Fourier basis. Then the states $\left|v^{(k)}\right\rangle=\sum_{m=0}^{d-1} v_{m}|(m+k) \bmod d\rangle$ form an orthonormal basis that is unbiased with respect to the Fourier basis.

Proof. First we prove that the unbiasedness of the vector $\left|v^{(0)}\right\rangle$ implies that all vectors $\left|v^{(k)}\right\rangle$ are unbiased to the Fourier basis. Indeed,

$$
\begin{aligned}
\left|\left\langle\tilde{J} \mid v^{(k)}\right\rangle\right|^{2} & =\left|\frac{1}{\sqrt{d}} \sum_{m=0}^{d-1} v_{m} \omega_{d}^{-j(m+k)}\right|^{2} \\
& =\left|\frac{1}{\sqrt{d}} \sum_{m=0}^{d-1} v_{m} \omega_{d}^{-j m}\right|^{2}=\frac{1}{d},
\end{aligned}
$$

where the step in between the lines follows from $\left|\omega_{d}^{-j k}\right|=$ 1 , and the last step expresses our assumption about the unbiasedness of the vector $\left|v^{(0)}\right\rangle$.

It remains to be shown that vectors $\left\{\left|v^{(k)}\right\rangle\right\}$ form a basis. To this end let us first compute their inverse Fourier transform:

$$
\begin{aligned}
\left|\tilde{v}^{(k)}\right\rangle & =F^{\dagger}\left|v^{(k)}\right\rangle=\frac{1}{\sqrt{d}} \sum_{l, m=0}^{d-1} v_{m} \omega_{d}^{-l(m+k)}|l\rangle \\
& =\sum_{l=0}^{d-1} \tilde{v}_{l} \omega_{d}^{-k l}|l\rangle,
\end{aligned}
$$

where we introduced $\tilde{v}_{l}=\frac{1}{\sqrt{d}} \sum_{m=0}^{d-1} v_{m} \omega_{d}^{-m l}$. Since the Fourier transform preserves the inner products, it is sufficient to show that the transformed vectors form a basis:

$$
\left\langle\tilde{v}^{(k)} \mid \tilde{v}^{\left(k^{\prime}\right)}\right\rangle=\sum_{l=0}^{d-1} \omega_{d}^{l\left(k-k^{\prime}\right)}\left|\tilde{v}_{l}\right|^{2}=\frac{1}{d} \sum_{l=0}^{d-1} \omega_{d}^{l\left(k-k^{\prime}\right)}=\delta_{k k^{\prime}}
$$

where, due to (5), we have used that $\left|\tilde{v}_{l}\right|^{2}=1 / d$ for all values of $l$.

Let us now restrict ourselves to prime dimensions $p$ which are equal to $p \equiv 3 \bmod 4$.

Lemma 2. Let $p$ be a prime with $p \equiv 3 \bmod 4$. Then the states $\left|v^{(k)}\right\rangle=\frac{1}{\sqrt{p}} \sum_{m=0}^{p-1} v_{m}|(m+k) \bmod p\rangle$ with

$$
v_{m}=\left\{\begin{aligned}
\frac{-(p-1)+2 \sqrt{-p}}{p+1} & \text { if } m \in \mathcal{Q}, \\
1 & \text { if } m \in \mathcal{N},
\end{aligned}\right.
$$

form a mutually unbiased basis with respect to both the standard and the Fourier bases. The sets $\mathcal{Q}$ and $\mathcal{N}$ denote the quadratic residues (including zero) and nonresidues modulo $p$, respectively.

Proof. Clearly, for all $m$ we have $\left|v_{m}\right|^{2}=1$, i.e., all vectors $\left|v^{(k)}\right\rangle$ are mutually unbiased with respect to the standard basis. We now prove that $\left|v^{(0)}\right\rangle$ is unbiased with respect to the Fourier basis. Consider

$$
\tilde{v}_{\tilde{J}}=\left\langle\tilde{J} \mid v^{(0)}\right\rangle=\frac{1}{p} \sum_{m=0}^{p-1} v_{m} \omega_{p}^{-j m} .
$$

For $j=0$ we find

$$
\begin{aligned}
\tilde{v}_{\tilde{0}} & =\frac{1}{p} \sum_{m=0}^{p-1} v_{m}=\frac{1}{p}\left(\frac{p-1}{2}+\frac{p+1}{2} \frac{2 \sqrt{-p}-(p-1)}{p+1}\right) \\
& =\frac{\sqrt{-p}}{p}
\end{aligned}
$$

where we have used the fact that the number of elements in $\mathcal{N}(\mathcal{Q})$ is equal to $\frac{p-1}{2}\left(\frac{p+1}{2}\right)$. This shows that indeed $\left|\tilde{v}_{\tilde{0}}\right|^{2}=$ $1 / p$. In order to find the other coefficients we use the Gauss 
period $\sum_{m \in \mathcal{Q}} \omega_{p}^{m}=(1+\sqrt{-p}) / 2$, when $p \equiv 3 \bmod 4$ [26], to show that for $j \not \equiv 0 \bmod p$ :

$$
\begin{gathered}
\sum_{m \in \mathcal{Q}} \omega_{p}^{-j m}=\frac{1-\sqrt{-p}}{2}, \\
\text { and } \sum_{m \in \mathcal{N}} \omega_{p}^{-j m}=\frac{-1+\sqrt{-p}}{2} .
\end{gathered}
$$

Thus, for $j \not \equiv 0 \bmod p$ we have

$$
\begin{aligned}
\tilde{v}_{\tilde{J}} & =\frac{1}{p}\left(\frac{-1+\sqrt{-p}}{2}+\frac{1-\sqrt{-p}}{2} \frac{2 \sqrt{-p}-(p-1)}{p+1}\right) \\
& =\frac{\sqrt{-p}}{p}
\end{aligned}
$$

revealing that $\left|v^{(0)}\right\rangle$ is unbiased to the Fourier basis. The proof concludes by utilizing Lemma 1 .

A concrete example of the basis introduced in Lemma 2 for dimension 7 reads as follows:

$$
A=\frac{1}{\sqrt{7}}\left(\begin{array}{ccccccc}
\alpha_{7} & \alpha_{7} & \alpha_{7} & 1 & \alpha_{7} & 1 & 1 \\
1 & \alpha_{7} & \alpha_{7} & \alpha_{7} & 1 & \alpha_{7} & 1 \\
1 & 1 & \alpha_{7} & \alpha_{7} & \alpha_{7} & 1 & \alpha_{7} \\
\alpha_{7} & 1 & 1 & \alpha_{7} & \alpha_{7} & \alpha_{7} & 1 \\
1 & \alpha_{7} & 1 & 1 & \alpha_{7} & \alpha_{7} & \alpha_{7} \\
\alpha_{7} & 1 & \alpha_{7} & 1 & 1 & \alpha_{7} & \alpha_{7} \\
\alpha_{7} & \alpha_{7} & 1 & \alpha_{7} & 1 & 1 & \alpha_{7}
\end{array}\right),
$$

where $\alpha_{7} \equiv \frac{\sqrt{-7}-3}{4}$. To see that the rows or columns correspond to orthogonal states note that $\alpha_{7}+\alpha_{7}^{*}=-\frac{3}{2}$.

We have shown that the standard basis, the Fourier basis, and the basis of Lemma 2 form a triple of MUBs. We verified by computer-aided techniques that this triple is unextendible in dimensions $p=7$ and $p=11$. We conjecture that for any prime $p$ with $p \equiv 3 \bmod 4$, the triple obtained in this way is unextendible. Note that in prime dimensions smaller than 7 , there are no unextendible sets of MUBs and, furthermore, the only existing sets of MUBs are maximal. From this perspective the existence of basis $A$ is unexpected.

\section{DIMENSION 13 AND PRIMES $p \equiv 1 \bmod 4$}

One can proceed along similar lines for other prime dimensions. This time the candidate basis is given by the following lemma.

Lemma 3. (See also Ref. [27].) Let $p$ be a prime with $p \equiv 1$ $\bmod 4$. Define

$$
z_{0}=\cos \theta+i \sin \theta \quad \text { with } \quad \cos \theta=\frac{\sqrt{p}-1}{p-1} .
$$

Then the states $\left|v^{(k)}\right\rangle=\frac{1}{\sqrt{p}} \sum_{m=0}^{p-1} v_{m}|(m+k) \bmod p\rangle$, where

$$
v_{m}= \begin{cases}1, & \text { if } m=0 \\ z_{0}, & \text { if } m \in \mathcal{Q} \backslash\{0\} \\ 1 / z_{0}, & \text { if } m \in \mathcal{N}\end{cases}
$$

form a mutually unbiased basis with respect to both the standard and the Fourier basis.

Proof. Since $z_{0}$ lies on the unit circle the vectors $\left|v^{(k)}\right\rangle$ are clearly mutually unbiased to the standard basis. Note also that $1 / z_{0}=z_{0}^{*}$, where $z_{0}^{*}$ denotes the complex conjugation of $z_{0}$.
Now we prove that $\left|v^{(0)}\right\rangle$ is unbiased with respect to the Fourier basis. For $j=0$ we have

$$
\begin{aligned}
\tilde{v}_{\tilde{0}} & =\frac{1}{p} \sum_{m=0}^{p-1} v_{m}=\frac{1}{p}\left(1+\frac{p-1}{2}\left(z_{0}+z_{0}^{*}\right)\right) \\
& =\frac{1}{\sqrt{p}},
\end{aligned}
$$

where we have used the fact that the sets $\mathcal{N}$ and $\mathcal{Q} \backslash\{0\}$ each have $\frac{p-1}{2}$ elements, and $z_{0}+z_{0}^{*}=2 \frac{\sqrt{p}-1}{p-1}$. To find the remaining coefficients we use the Gauss period $\sum_{m \in \mathcal{Q}} \omega_{p}^{m}=$ $(\sqrt{p}+1) / 2$, when $p \equiv 1 \bmod 4$ [26], to derive the following two equations:

$$
\begin{gathered}
\sum_{m \in \mathcal{Q} \backslash\{0\}} \omega_{p}^{-j m}=\frac{\sqrt{p}-1}{2}, \\
\sum_{m \in \mathcal{N}} \omega_{p}^{-j m}=\frac{-\sqrt{p}-1}{2},
\end{gathered}
$$

which apply for $j \not \equiv 0 \bmod p$. Therefore, for $j \not \equiv 0 \bmod p$,

$$
\tilde{v}_{\tilde{\jmath}}=\frac{1}{p}\left(1+\frac{\sqrt{p}-1}{2} z_{0}+\frac{-\sqrt{p}-1}{2} z_{0}^{*}\right),
$$

and hence,

$$
\begin{aligned}
\left|\tilde{v}_{\tilde{J}}\right|^{2} & =\frac{1}{p^{2}}\left|1-\frac{1}{2}\left(z_{0}+z_{0}^{*}\right)+\frac{\sqrt{p}}{2}\left(z_{0}-z_{0}^{*}\right)\right|^{2} \\
& =\frac{1}{p^{2}}\left(2-2 \cos \theta+(p-1)\left(1-\cos ^{2} \theta\right)\right) \\
& =\frac{1}{p}
\end{aligned}
$$

Thus, the vector $\left|v^{(0)}\right\rangle$ is unbiased to the Fourier basis, and by application of Lemma 1 we have completed the proof.

Again we have verified by computer-aided algebraic methods that this set is unextendible in dimension $p=13$, and conjecture that it is unextendible in all prime dimensions $p \equiv 1$ mod 4. In this way we have now covered all prime dimensions.

\section{COMPOSITE DIMENSIONS}

In a composite dimension $d=p_{1}^{e_{1}} \cdots p_{m}^{e_{m}}$ (with prime factors $p_{i} \neq p_{j}$ for $i \neq j$ ), most of our small sets of complementary observables follow from the results in Ref. [28]. It is useful to introduce the Weyl-Heisenberg operators

$$
\begin{gathered}
Z_{d}|j\rangle=\omega_{d}^{j}|j\rangle, \\
X_{d}|j\rangle=|(j+1) \bmod d\rangle .
\end{gathered}
$$

It turns out that there always exists a set of

$$
\xi=\min _{i} p_{i}+1
$$

complementary observables corresponding to the eigenbases of the operators

$$
Z_{d}, X_{d}, X_{d} Z_{d}, X_{d} Z_{d}^{2}, \ldots, X_{d} Z_{d}^{\xi-2}
$$

that is "weakly" unextendible in the sense that it cannot be extended by another basis which is an eigenbasis of any 
other Weyl-Heisenberg operator. They are therefore natural candidates for truly unextendible bases. Indeed, we verified for all even composite dimensions presented in Table I that the three eigenbases of $Z_{d}, X_{d}$, and $X_{d} Z_{d}$ are strongly unextendible. It is therefore reasonable to conjecture in general that the complementary observables (25) form an unextendible set.

In dimensions 9 and 15 the bound of Eq. (24) gives four bases, but we found unextendible sets with only three measurements. These sets include the standard basis, its Fourier transform, and a third basis. However, as it is difficult to recognize the structure of the third bases, we will not report them here explicitly.

\section{CONTINUOUS VARIABLES}

One can generalize the notion of mutually unbiased bases to an infinite dimensional Hilbert space by defining two bases $\{|\phi\rangle\}$ and $\{|\psi\rangle\}$ as mutually unbiased if $|\langle\phi \mid \psi\rangle|^{2}=\kappa$, for all vectors $|\phi\rangle$ and $|\psi\rangle$, with $\kappa$ a positive constant. Here we also assume that both bases satisfy the orthonormality condition in terms of a Dirac $\delta$, i.e., $\left\langle\psi \mid \psi^{\prime}\right\rangle=\delta\left(\psi-\psi^{\prime}\right)$ and $\left\langle\phi \mid \phi^{\prime}\right\rangle=$ $\delta\left(\phi-\phi^{\prime}\right)$.

For an example, consider the Hilbert space $L^{2}(\mathbb{R})$ and the generalized eigenstates $\left|q_{\theta}\right\rangle$ of the operator $\hat{q}_{\theta}=\cos \theta \hat{q}+$ $\sin \theta \hat{p}$, where $\hat{q}$ and $\hat{p}$ are the position and momentum operators, respectively. The overlap of $\left|q_{\theta}\right\rangle$ with $\left|q_{\theta^{\prime}}\right\rangle$ can be calculated from the Wigner functions of the quantum states and is given by [29]:

$$
\left|\left\langle q_{\theta} \mid q_{\theta^{\prime}}\right\rangle\right|^{2}=\frac{1}{2 \pi \hbar\left|\sin \left(\theta-\theta^{\prime}\right)\right|} .
$$

Since the overlap depends only on the angles $\theta$ and $\theta^{\prime}$, the bases $\left\{\left|q_{\theta}\right\rangle\right\}$ and $\left\{\left|q_{\theta^{\prime}}\right\rangle\right\}$ are mutually unbiased. By fixing the first basis to be $\{|q\rangle\}$, with $\theta=0$, one can find a symmetric triple of MUBs [30]: $\{|q\rangle\},\left\{\left|q_{+}\right\rangle\right\}$, and $\left\{\left|q_{-}\right\rangle\right\}$, where $\hat{q}_{ \pm}=$ $\cos (2 \pi / 3) \hat{q} \pm \sin (2 \pi / 3) \hat{p}$.

It is straightforward to see from the Wigner functions that multiplying the operator $\hat{q}_{\theta^{\prime}}$ by $r$ results in the overlap $\left|\left\langle q_{\theta} \mid r q_{\theta^{\prime}}\right\rangle\right|^{2}=1 / 2 \pi \hbar r\left|\sin \left(\theta-\theta^{\prime}\right)\right|$. We now show that by considering eigenstates of operators of the form $r \hat{q}_{\theta}$, it is always possible to extend any pair of MUBs to a triple of MUBs.

Lemma 4. If we consider MUBs from the eigenstates of observables $r \hat{q}_{\theta}$, then no unextendible pair of MUBs exists, and all triples are unextendible.

Proof. We rotate the coordinate system and set the unit of length such that for the first operator we have $\theta=0$ and $r=1$. With this choice, we now show that MUBs corresponding to the operators $\hat{q}$ and $r \hat{q}_{\theta}=r(\cos \theta \hat{q}+\sin \theta \hat{p})$ always extend to a third MUB for all $\theta \in(0,2 \pi), \theta \neq \pi$, and $r>0$. We consider a third operator $s \hat{q}_{\phi}$ and show that the mutual unbiasedness equations

$$
r|\sin \theta|=s|\sin \phi|=r s|\sin (\theta-\phi)|
$$

are satisfied for a particular choice of $s$ and $\phi$. Choosing $s=$ $\frac{r|\sin \theta|}{|\sin \phi|}$, the first equality is satisfied for all $\theta, \phi \neq \pi$, and $r$. The final condition becomes $|\sin \phi|=r|\sin (\theta-\phi)|$, which is satisfied when

$$
\phi_{ \pm}=\arctan \left(\frac{ \pm r \sin \theta}{1 \pm r \cos \theta}\right) .
$$

Hence, the operators $\left(\hat{q}, r \hat{q}_{\theta}, s_{ \pm} \hat{q}_{\phi_{ \pm}}\right)$with $s_{ \pm}=\frac{r|\sin \theta|}{\sin \left(\phi_{ \pm}\right)}$and $\phi_{ \pm}$ in Eq. (28) correspond to two sets of three MUBs, each with two parameters. If we consider a fourth basis corresponding to the operator $t \hat{q}_{\nu}$, it is easy to check that there is no mutually unbiased set of four bases.

For example, choosing $r=1$ and $\theta=2 \pi / 3$ we arrive at two triples, the symmetric mutually unbiased triple $\left(\hat{q}, \hat{q}_{2 \pi / 3}, \hat{q}_{4 \pi / 3}\right)$, and $\left(\hat{q}, \hat{q}_{2 \pi / 3}, \sqrt{3} \hat{q}_{\pi / 6}\right)$. Choosing $r=1$ and $\theta=\pi / 2$ we find the asymmetric triples $\left(\hat{q}, \hat{p}, \sqrt{2} \hat{q}_{ \pm \pi / 4}\right)[30]$.

\section{APPROACHES TO UNEXTENDIBILITY}

Here we discuss two approaches which reveal that unextendible MUBs are present in infinitely many dimensions. A related result can be found in Refs. $[9,10]$, but our analysis extends to other dimensions.

\section{A. Real Hadamards}

Both of our approaches utilize a result which limits the number of real bases in a complete set of MUBs [31]. In particular, given a complete set of MUBs in dimension $d$, written in the form $\left\{\mathbb{1}, H_{1}, \ldots, H_{d}\right\}$, where $\mathbb{1}$ is the identity matrix and $H_{i}$ are complex Hadamard matrices of order $d$, then at most one of the Hadamard matrices $H_{i}$ can be real. Therefore, any set of complementary observables represented by the identity and at least two real Hadamard matrices is part of an unextendible set.

In dimensions $d=4^{i}, \quad$ constructions given in Refs. [3,32,33] find $d / 2+1$ real MUBs (in some of these dimensions Ref. [34] finds a smaller number of real MUBs but still larger than 3). These bases can be transformed into a set of $d / 2$ real Hadamard matrices, which therefore belong to an unextendible set of MUBs. While no additional real basis is mutually unbiased to this set, $d / 2+1$ gives only a lower bound on the cardinality of the unextendible set. Other complex Hadamard matrices may exist which represent additional MUBs with respect to the whole set.

\section{B. Mutually orthogonal Latin squares}

A better lower bound is achieved, and for a larger set of dimensions, by starting with mutually orthogonal Latin squares (MOLSs). Reference [35] describes a procedure using Hadamard matrices and MOLSs, which transforms a set of $m$ squares of order $d$ to a set of $m+2$ MUBs in dimension $d^{2}$. Bachelor Latin squares are squares which have no orthogonal mate (i.e., an unextendible set of MOLSs with $m=1$ ) and have recently been shown to exist for all $d>3$ [36,37]. We therefore use the construction of [35] with a bachelor Latin square and a real Hadamard matrix of order $d$. The Hadamard conjecture suggests that such a matrix always exists for all $d=4 s$, and according to most recent checks, the first case in which a real Hadamard matrix has not yet been found is $s=668$ [38]. With these ingredients the procedure yields three real MUBs $\left\{B_{1}, B_{2}, B_{3}\right\}$. To transform this set to the standard form, we multiply each matrix with the transposed 
matrix $B_{1}^{T}$, i.e., $\left\{\mathbb{1}, B_{2} B_{1}^{T}, B_{3} B_{1}^{T}\right\}$. The matrices $B_{2} B_{1}^{T}$ and $B_{3} B_{1}^{T}$ are now two real Hadamard matrices, and the triple of MUBs does not extend to a complete set. Unfortunately, this lower bound is not tight. We have verified in dimension $d=16$ that the three MUBs obtained in this way can be extended to at least five bases.

Finally, we utilize the concept of unextendibility to comment briefly on any conjectured strong relationship between MOLSs and MUBs. It has been observed that certain complete sets of MOLSs translate into complete sets of MUBs in prime and prime-power dimensions via an algorithm given in Ref. [4]. Similarly, in a reverse process, one can construct complete sets of MOLSs from certain complete sets of MUBs [39]. It might therefore be tempting to conjecture a strong statement that to every set of MOLSs there corresponds a set of MUBs. For the algorithm given in Ref. [4] this is disproved in Ref. [40]. Here we note that such a conjecture cannot be true for any algorithm directly linking sets of MOLSs and MUBs, and preserving (un)extendibility. This follows from the observation that a bachelor Latin square should correspond to a set of three unextendible MUBs in any dimension $d>3$. However, this is not the case for dimension 5 where no unextendible set of MUBs exists (cf. Sec. III). We finish by noting that the weaker statement given in Ref. [41], that a maximal set of MUBs exists in those dimensions $d$ for which one can find a maximal set of MOLSs of order $d$, might still hold true.

\section{CONCLUSIONS}

We presented small and unextendible sets of complementary observables, usually containing three measurements. It is of course interesting to ask if even smaller sets exist with the ultimate limit of two. Mathematically these would correspond to so-called bachelor Hadamard matrices, which so far are only known to exist in dimension 6 . We therefore hope our study will motivate the search for bachelor Hadamards in other dimensions as well as the search for further applications of unextendible MUBs.

\section{ACKNOWLEDGMENTS}

We thank Łukasz Rudnicki for pointing out that unextendible MUBs can distinguish some mixed states from pure states. This work is supported by the National Research Foundation and Singapore Ministry of Education Academic Research Fund Tier 2 Project No. MOE2015-T2-2-034. D.M. acknowledges support by the Institute for Information \& Communications Technology Promotion (IITP) Grant funded by the Korean government (MSIP) (No. R0190-16-2028, PSQKD).
[1] T. Durt, B.-G. Englert, I. Bengtsson, and K. Życzkowski, Int. J. Quant. Inf. 08, 535 (2010).

[2] M. Grassl, in Proc. ERATO Conf. on Quantum Information Science 2004 (EQIS 2004), arXiv:quant-ph/0406175 (2004).

[3] P. Boykin, M. Sitharam, M. Tarifi, and P. Wocjan, arXiv:quant$\mathrm{ph} / 0502024$.

[4] T. Paterek, B. Dakić, and Č. Brukner, Phys. Rev. A 79, 012109 (2009).

[5] S. Brierley and S. Weigert, Phys. Rev. A 79, 052316 (2009).

[6] P. Jaming, M. Matolcsi, P. Móra, F. Szöllősi, and M. Weiner, J. Phys. A 42, 245305 (2009).

[7] D. McNulty and S. Weigert, J. Phys. A 45, 102001 (2012).

[8] D. Goyeneche, J. Phys. A 46, 105301 (2013).

[9] A. Szanto, L. Alg. Appl. 496, 392 (2016).

[10] J. Jedwab and L. Yen, arXiv:1604.04797 [math.CO].

[11] P. Mandayam, S. Bandyopadhyay, M. Grassl, and W. K. Wootters, Quant. Inf. Comp. 14, 823 (2014).

[12] K. Thas, Entropy 18, 395 (2016).

[13] V. Hegde and P. Mandayam, arXiv:1508.05892 [quant-ph].

[14] J. Schwinger, Proc. Nat. Acad. Sci. USA 46, 570 (1960).

[15] K. Korzekwa, D. Jennings, and T. Rudolph, Phys. Rev. A 89, 052108 (2014).

[16] O. Andersson and I. Bengtsson, arXiv:1506.09062 [quant-ph].

[17] L. Jakóbczyk and M. Siennicki, Phys. Lett. A 286, 383 (2001).

[18] G. Kimura, Phys. Lett. A 314, 339 (2003).

[19] D. M. Appleby, in SIC-POVMS and MUBS: Geometrical Relationships in Prime Dimensions, AIP Conf. Proc. No. 1101 (AIP, Melville, NY, 2009), p. 223.

[20] C. H. Bennett, D. P. DiVincenzo, T. Mor, P. W. Shor, J. A. Smolin, and B. M. Terhal, Phys. Rev. Lett. 82, 5385 (1999).

[21] S. Bravyi and J. A. Smolin, Phys. Rev. A 84, 042306 (2011).
[22] S. Brierley, S. Weigert, and I. Bengtsson, Quant. Inf. Comp. 10, 803 (2010).

[23] M. Weiner, Proc. Amer. Math. Soc. 141, 1963 (2013).

[24] U. Haagerup, in Operator Algebras and Quantum Field Theory edited by S. Doplicher et al. (International Press, Cambridge, MA, 1997).

[25] A. T. Butson, Proc. Amer. Math. Soc. 13, 894 (1962).

[26] H. Davenport and H. L. Montgomery, Multiplicative Number Theory (Springer-Verlag, Berlin, Heidelberg, New York, 1980).

[27] E. M. Gabidulin and V. V. Shorin, Probl. Inf. Trans. 38, 255 (2002).

[28] M. Aschbacher, A. M. Childs, and P. Wocjan, J. Alg. Comb. 25, 111 (2007).

[29] K. S. Gibbons, M. J. Hoffman, and W. K. Wootters, Phys. Rev. A 70, 062101 (2004).

[30] S. Weigert and M. Wilkinson, Phys. Rev. A 78, 020303 (2008).

[31] M. Matolcsi, I. Z. Ruzsa, and M. Weiner, Australas. J. Combin. 55, 35 (2013).

[32] P. J. Cameron and J. J. Seidel, Indag. Math. 76, 1 (1973).

[33] A. R. Calderbank, P. J. Cameron, W. M. Kantor, and J. J. Seidel, Proc. London Math. Soc. 75, 436 (1997).

[34] R. Gow, arXiv:1410.4059 [math.GR].

[35] P. Wocjan and T. Beth, Quant. Inf. Comp. 5, 93 (2005).

[36] A. B. Evans, Des. Codes. Crypt. 40, 121 (2006).

[37] I. M. Wanless and B. S. Webb, Des. Codes. Crypt. 40, 131 (2006).

[38] H. Kharaghani and B. Tayfeh-Rezaie, J. Combin. Des. 13, 435 (2005).

[39] J. L. Hall and A. Rao, J. Phys. A 43, 135302 (2010).

[40] T. Paterek, M. Pawłowski, M. Grassl, and Č. Brukner, Phys. Scr. T140, 014031 (2010).

[41] M. Saniga, M. Planat, and H. Rosu, J. Opt. B 6, L19 (2004). 